\title{
Enfoque científico y filosófico de la aplicación de un modelo de costeo ABC como alternativa de desarrollo del sector agrícola ecuatoriano
}

\section{Scientific and philosophical approach to the application of an ABC costing model as an alternative for the development of the Ecuadorian agricultural sector}

Erika Sonia Quiñonez Alvarado

https://orcid.org/0000-0002-3520-5183

Universidad de Guayaquil, Ecuador

Autor por correspondencia: erika.quinoneza@ug.edu.ec

Fecha de recepción: 13 de noviembre de 2019 - Fecha de aceptación: 20 de abril de 2020

Resumen

Este estudio tiene como finalidad explicar la concepción del conocimiento, filosofía y ciencias que se considera adecuada para la propuesta de aplicación de un modelo de costeo $\mathrm{ABC}$ como alternativa viable para el desarrollo del sector agrícola rural ecuatoriano, se recurrió a la revisión de textos de varios filósofos afines a la ideología de las ciencias contables, para conocer sus teorías, enfoques epistemológicos y metodologías para luego de su entendimiento relacionarlos con el tema planteado. Desde el punto de vista de lo que se desea lograr con esta propuesta, se evidencia que la concepción de la investigación se dirige a la corriente positivista mientras que el desarrollo del modelo se considera debe ser demostrado en falso para evitar contingencias. Se concluye que a partir de la investigación científica se pueden generar teorías contables que luego de ser validadas pueden ser aceptadas por la sociedad y así darle el reconocimiento a la Contabilidad como Ciencia.

Palabras claves: contabilidad; disciplina; modelos heurísticos; positivismo; falsacionismo

\begin{abstract}
The purpose of this study is to explain the conception of knowledge, philosophy and sciences that is considered adequate for the proposal to apply an ABC costing model as a viable alternative for the development of the Ecuadorian rural agricultural sector. Philosophers related to the ideology of the accounting sciences, to know their theories, epistemological approaches and methodologies, and after their understanding, relate them to the issue raised. From the point of view of what you want to achieve with this proposal, it is evident that the conception of the investigation is directed at the positivist current, while the development of the model is considered to be falsely demonstrated to avoid contingencies. It is concluded that from scientific research, accounting theories can be generated that after being validated can be accepted by society and thus give recognition to Accounting as Science.
\end{abstract}

Key words: accounting; discipline; heuristic models; positivism; falsificationism 


\section{Introducción}

Este ensayo presenta un análisis de la concepción científica y filosófica que se pretende aplicar al proponer un sistema de costeo ABC basado en actividades para el desarrollo del sector agrícola ecuatoriano, con el fin de promover el impulso e intervención de este conglomerado como actor principal en el ámbito comercial y económico del país, ya que actualmente pese a su aporte a la actividad agroalimentaria, atraviesa grandes dificultades como "altos costos de producción, normativas cambiantes, regulaciones laborales incompatibles" (Gómez, 2019). Reflexionado la importancia de esta comunidad en la seguridad alimentaria local; el respeto a las practicas ancestrales amigables con el medio ambiente, y a la tendencia global del consumo de alimentos orgánicos que propenden al bienestar general.

Actualmente, existe debate sobre si la contabilidad debe ser considerada una ciencia esto se debe al diseño curricular que limita la investigación en los planes de estudio del perfil profesional que contribuyan al desarrollo científico de la misma, sin embargo, esto puede ser cambiado a través de los planes doctorales que se están promoviendo actualmente. Si bien no es parte de este ensayo entrar en esta controversia es necesario exponerlo para ver el enlace de la Contabilidad como disciplina que se enmarca en lo social sustentada en la economía neoclásica orientada a la teoría contable aplicada al tema propuesto como un aporte científico que ayude a la colectividad rural agrícola a su inclusión protagónica en el entorno económico del Ecuador.

Este articulo tiene como enfoque de investigación el fortalecimiento de los actores de la economía y desarrollo empresarial del sector agrícola rural ecuatoriano, quienes a lo largo del tiempo han desempeñado un papel preponderante en el contexto de seguridad alimentaria del país, más su aporte no ha sido retribuido en la medida de su contribución, se plantea como con herramientas cimentadas en la contabilidad se pueden crear propuestas pertinentes que propendan al desarrollo económico y social de la comunidad objeto de estudio.

En cuanto a la filosofía se busca relacionar el objeto de estudio del proyecto de tesis doctoral planteado "Diseño de un Modelo de costos basados en actividades para el desarrollo del productor agrícola rural ecuatoriano" y el enfoque positivista de Augusto Comte, puesto que esta se "funda en la ciencia" (Comte, 1875, p. 12) aplicando la teoría del conocimiento basados en valores de orden y progreso, al catalizar el desarrollo del sector agrícola de Ecuador se requiere estudiar los procesos que involucran la producción de alimentos comprendida en su planificación, cultivo, cosecha y comercialización como una perspectiva para el crecimiento económico de esta comunidad orientadas hacia una producción sustentable.

Se requiere estudiar las personas, infraestructura, recursos, contexto, perspectiva futura, políticas públicas desde el punto de vista de la disciplina contable para incorporar al agricultor rural a la sociedad del conocimiento. Con el fin de diseñar un modelo de costeo pertinente para el agricultor rural ecuatoriano que le permita a través de la validación la optimización de recursos para una gestión exitosa e integra que transforme la situación precaria en la que actualmente se encuentra y su actividad pueda responder no únicamente a las demandas alimentarias del país sino de países que demandan producción certificada como orgánica. 
Para demostrar la concepción del conocimiento científico y la postura filosófica acogida por el autor en este documento, se hará una revisión bibliográfica de los núcleos teóricos expuestos para enlazarlos con la investigación y la ciencia, las ramas de la filosofía y la aproximación al positivismo Comtiano, esto es interrelacionar lo filosófico y científico. Se responde a la pregunta ¿Por qué adoptar el positivismo como corriente filosófica para este proyecto? Se analiza y se discute sobre las filosofías contemporáneas abordadas en el curso "Filosofía de las Ciencias Contables" por el Dr. Víctor Baltodano Azabache, otros pensadores que tienden a la filosofía positivista; se realiza también un análisis de las oposiciones al positivismo conocido como el falsacionismo autores destacados del siglo XX que formulan sus propios métodos y que forman parte de la bibliografía usada en este ensayo.

Se establece como hipótesis del proyecto de tesis "el diseño de un método de costeo por actividades pertinente para los agricultores rurales ecuatorianos, les permitirá desarrollar al sector agrícola", misma que si bien no se probara en este ensayo se busca justificar de manera científica y filosófica.

El presente trabajo contiene además el desarrollo de la temática donde se hace un discernimiento científico y filosófico de los núcleos teóricos que rodean el tema, además las conclusiones donde se contrasta los puntos de vista tratados y finalmente los referentes bibliográficos.

\section{Análisis de la concepción del conocimiento científico y filosofía para el proyecto de investigación}

\section{Concepción científica para el proyecto de investigación}

Según Goode \& Hatt (1975) Ciencia es un método de acercamiento a todo el mundo empírico, es decir susceptible de ser sometido a experiencias. En el ámbito moderno se exige la relación entre la teoría y el hecho, considerando que de manera individual la perspectiva varía entre uno y otro. Este pensamiento tiene relación con el de Baltodano (2010) quien usó el concepto de método científico conformacional para la producción de nuevas existencias y explica que es necesario esclarecer el método de las ciencias para los pueblos que no la producen por diversos factores de su contexto social. (p.357). Es decir, que la ciencia debe conformarse por todos los individuos pues la ciencia no es excluyente.

Debido a sus características la propuesta planteada se ubica en la disciplina contable. De acuerdo con los postulados concebidos por Khun (1962), la Contabilidad aún no se configura como ciencia normal, puesto que para lograr ser considerada como tal el paradigma exige de manera irrestricta a los científicos en este caso los contadores a investigar su naturaleza de una forma minuciosa y honda que sería inimaginable en otras condiciones, y que sustente el modo de ser y de pensar del entorno donde se aplica. Pero ¿qué son los paradigmas?, el autor los define como realizaciones científicas o verdades innegables reconocidas globalmente en el contexto científico.

Si bien la ciencia contable tiene su origen miles de años atrás y ha presentado una evolución teórica y metodológica, aún no repercute significativamente en el contexto científico, es decir ser generalizada por una colectividad de investigadores contadores. Sin embargo, es una disciplina emergente cuya comunidad académica va en auge orientada a la teoría contable. La integración de un objeto de estudio específico y la comunidad científica con intereses afines, es decir una aceptación general, es el factor preponderante para su

Esta obra se comparte bajo la licencia Creative Common Atribución-No Comercial 4.0 International (CC BY-NC 4.0) 
reconocimiento como ciencia. (Montes, Soto, \& Valencia, 2006) Más, es una disciplina sostenida en teorías sociológicas que abarca modelos heurísticos, dos componentes esenciales de un paradigma según la tesis de (Khuhn, 1962).

La teoría epistemológica conformacional se sintetiza en tres perspectivas la identificación diagnóstica de los contenidos, la desestructuración de y desestructuración conceptual y la propuesta conformacional para la creación de teorías para la producción de conocimientos. (Baltodano, 2010).

La disciplina contable es la sistematización de conocimientos, producto de la sociedad al servicio de la misma para el beneficio de sus miembros; coordina y dirige la actividad de resolver problemas; gira alrededor del patrimonio; $y$, formula juicios y razonamientos coherentes, considerándose de acuerdo a Torres una ciencia aplicada en vías de maduración. (2012) Se fundamenta en preceptos de contabilidad de general aceptación y se rige por el principio de la partida doble.

La contabilidad desde el punto de vista social se sustenta en las teorías: Neoclásica, beneficio verdadero, utilidad, institucional, participe, legitimación, constructivista, crítica. Es decir, se considera que la información que proporciona debe tener efectos sociales y medioambientales. La teoría neoclásica está orientada al valor de los bienes acorde con el bienestar de los usuarios. La teoría constructivista se basa en la enseñanza-aprendizaje no excluyente. La teoría crítica aspira una comprensión histórico-cultural de la sociedad. (Diaz, 2003).

A partir de la perspectiva que la contabilidad abarca modelos heurísticos que según la enunciación de Khun (1962), son modelos típicos de resolución de problemas coherentes con el desarrollo ontológico es decir del ser o ente (p. 75), es claro que una herramienta contable como un modelo de costeo por actividades ABC pertinente con las necesidades auténticas del sector rural se enmarca en la concepción científica apoyada en las bases teóricas y conceptuales aquí descritas, y que la contabilidad por su compromiso con el bienestar común se encuentra en el camino de ser considerada una ciencia.

Donde la optimización de los recursos resulte en beneficios para los partícipes del ciclo económico esto es: el agricultor rural, el consumidor y por ende la colectividad por la aplicación de prácticas agrícolas ancestrales reconocidas como medioambientales en la parte social y por la activación de la matriz productiva en el ámbito económico. Se cumple el precepto de la solución de un problema como es la falta de incentivo al campesino, el reconocimiento de nuestra herencia ancestral a respetar la naturaleza, el beneficio al consumidor con productos orgánicos que coadyuvan a la salud, y la sostenibilidad alimentaria y económica de todo el país. Esto sustentado en el conocimiento, la experimentación y validación de la ciencia.

\section{Enfoque filosófico adoptado para el "Diseño de un Modelo de costos basados en actividades para el desarrollo del productor agrícola rural ecuatoriano"}

En el contexto filosófico contemporáneo se destacan según el Dr. Víctor Baltodano, (2010) corriente tales como el positivismo, espiritualismo, existencialismo, pragmatismo, estructuralismo, materialismo dialéctico, posmodernismo, pensamiento complejo y filosofía conformacional. Para identificar cual acoger para el proyecto de tesis propuesto es necesario analizar cada uno de ellos y luego contrastar con autores que han aportado o rechazado los 
postulados de la filosofía adoptada de acuerdo a sus propios textos y al libro guía de reflexión filosófica del Dr. Baltodano.

El positivismo de August Comte sostuvo que el conocimiento verdadero se obtiene mediante la ciencia. Cualquier disciplina debe validarse en positivo usando reglas de validación coherentes, la razón prevalece; en función de los hechos, las leyes que rigen los fenómenos son comprobados por métodos científico empírico de la observación o experiencia personal, para evaluar su implicación posterior, "ver para prever". (1875).

Si bien Comte es reconocido como el pionero del positivismo existen otros filósofos que han profundizado y depurado esta corriente se denomina neopositivismo es el caso de Ernst Mach, quien sostuvo que las verdaderas leyes que rigen al mundo son las sensaciones, consideraba "una cosa es un símbolo mental de una sensación compuesta de relativa fijeza" Es decir la ciencia se trata de fenómenos, es decir los hechos como se manifiestan en las experiencias, consideró que el fin de la ciencia es la limitación del pensamiento.

Por su lado Moritz Schlick, filósofo y científico austriaco, fundador del Círculo de Viena, declaró que un enunciado no comprobado científicamente no tiene sentido, introduce el proceso de contratación y demostración en base a fundamentos neopositivistas. Además, expresó que el investigador debe ir más allá a fin de constatar la experiencia. Tanto positivismo como neopositivismo coinciden en la verificación de los hechos en positivo aplicando métodos científicos empíricos. (Atencia, 1991) es decir, son estudios confirmatorios que parten de una premisa o hipótesis. El conocimiento científico debe ser validado no atribuido meramente, los hechos son comprobables, cuantificables y se pueden explicar de manera técnica y práctica.

La corriente filosófica del existencialismo según Martín Heidegger en su obra Ser y tiempo (1993) analizó la existencia humana, es crítico en sus concepciones, expresa que el hombre se hace a partir las experiencias y vivencias propias son sus decisiones que lo definen, expone que la muerte es una situación implícita de la existencia que limita a la concreción de todas las metas, somos finitos. El conocimiento del mundo se entiende como una relación entre sujeto y objeto, aunque no coinciden con Dasein (ontológicamente a si mismo) y mundo. En ser y tiempo reflexiona que el hombre abandona el ser para consagrarse al dominio de las cosas o materialismo y el tiempo se establece en presente, pasado y futuro. Este razonamiento está presente actualmente de manera generalizada no solo en la generación de conocimiento sino la vida misma, vivimos como elegimos vivir, excepto la muerte no está a elección, pero es una realidad en limitante y abrupto, se vive de prisa y dejamos de lado lo importante por lo material en una era donde la tecnológica nos acerca a las tendencias globales.

El estructuralismo de Piaget, definió que el desarrollo de la teoría cognitiva experimentada por el ser humano se da en cuatro estadios de aprendizaje que corre de cero a dos años la sensorial motriz, de dos a siete años pre operacional, de siete a doce años operacional concreto, y en la adolescencia el operacional formal. Luego el avance del intelecto es la combinación de las experiencias previas y nuevas, si son paralelas se denomina equilibrio cognitivo cuando son discordante crean confusión en el individuo al inicio para luego transformarse en aprendizaje. Para la concepción del hombre y la sociedad los estructuralistas al estudiar la realidad histórica usan un conjunto ordenado de instrumentos intelectuales operativos y así definen estructura. Empiezan describiendo y explicando elementos, aspectos y formas sociales y luego las comparan, simplificando los 
elementos y procesos en modelos, el trabajo intelectual está basado y procesado en una operación metalingüística que tiene relación con lo descrito. Coinciden con el positivismo en sostener que la realidad existe en tanto pueda ser percibida. (Baltodano, 2010).

Esta corriente filosófica ayuda a describir de manera cronológica los pasos de la investigación desde su origen hasta su resultado, analizar la conducta de los individuos a partir de las cuales se pueden generar alternativas proyectadas en torno a las leyes universales de manera operacional.

El espiritualismo concepción filosófica de acuerdo a la percepción de Santo Tomas de Aquino se desprende de Dios creador, que rige el universo mediante la ley eterna o divina separa a los seres naturales carentes de conocimientos regidos por las leyes físicas, del ser humano racional por tanto regido por la ley natural o moral dirigida a la conducta, aborda preceptos tales como deber de conservación de la vida y la naturaleza, la procreación, vivir en sociedad y buscar la justicia en la vida social "debe hacerse el bien y evitarse el mal" estas percepciones son reguladas por la ley que crean los humanos concordante a la universal y esta se denomina positiva. Sostiene además que toda causa es consecuencia de otra causa esta. (Alarcón, 2014) es decir, Tomas Aquino reconoce capacidades intelectuales en el hombre para discernir sobre la ética.

El pragmatismo es considerado una pedagogía activa creada por John Dewey "aprender haciendo", el filósofo William James es otro exponente de esta corriente con su teoría de la "verdad" se comprueba el pensamiento por medio de la acción para convertirlo en conocimiento, al igual que el positivismo se aprende de las experiencias busca mejorar la calidad de vida de los seres humanos dando énfasis a la transformación social para su bienestar. La practicidad se considera acción relevante para sustentar el aprendizaje convirtiéndolo en una corriente activa donde el docente es el conductor para el logro de resolución del accionar práctico. (Barton, 2018) Al igual que las anteriores corrientes de la filosofía converge en el positivismo pues busca mejorar las condiciones de la sociedad por la inclusión justa de esta al conocimiento. Conocimiento que se genera por la experiencia en este caso usando la práctica para lograr el aprendizaje. Considerando que la verdad siempre será positiva por su concepción.

El materialismo dialéctico agrupa a varios filósofos reconocidos entre ellos Hegel y Marx esta corriente se divide en tres estadios: la era mecanicista siglo XVIII todo está en movimiento pero no se conoce la historia, sostenía que el medio es producto del hombre no existe evolución; el de la era metafísica donde la ciencia es estática las cosas son como las percibimos prevalece el capitalismo egoísta, se separa la ciencia de la filosofía y la política, es excluyente; la era dialéctica de Marx como método de conocimiento guía de acción para prever el futuro se enfoca la investigación en combinación con la practica el capitalismo es una realidad pero no definitivo ha surgido de un proceso que puede no ser permanente, se debe estudiar el origen de las cosas y como estas cambian porque cada objeto tiene su contradicción, se creía lo idealista. (Spirkin, 1969).

Las teorías filosóficas de Marx están subyacentes en el desarrollo de los aspectos económicos y sociales a nivel global, su influencia en las investigaciones dirigidas a mejorar la calidad de vida de un sector desprotegido entra en las concepciones del socialismo y es positiva porque el bienestar que se procura se lo verificará en positivo y se planifica para el futuro. 
La posmodernidad es el resultado de la modernidad la nombró así Lyotard, (1994) como consecuencia del fracaso de los resultados de las corrientes modernas y contemporáneas, comenzó en el siglo XX se caracteriza por las libertades, critica la modernidad porque el progreso ha fracasado, el pensamiento se orienta a la no resolución de las crisis pero si se puede disminuir a través de cambios. Presenta una postura realista considerada como intelectual, se respeta el punto de vista individual se elimina la concepción del bien y el mal, porque todo depende de la perspectiva del individuo, es netamente positiva en su confirmación se basa solo en hechos y busca la realización personal.

La filosofía conformacional explicada por el filósofo Baltodano, (2010) en su obra relaciona varios conceptos filosóficos de otras corrientes aquí descritas para concretarlos en una forma de conocimiento general que abarca desde lo abstracto o mitológico con lo estructural, donde se discierne el bien y el mal para configurar la sistematización de la razón, la filosofía y la ciencia aplicada a los países latinoamericanos que tradicionalmente no desarrollan ciencia propia, rompiendo la barrera para impulsar a los pueblos que hasta ahora se encontraban en estado de dependencia a estructurar pensamientos que dada la forma conceptual y comprensiva puedan ser vistos como creadores e inventores de conocimientos. Al ser una filosofía incluyente para la generación de conocimiento donde se sincretiza muchas otras corrientes esta debe ser comprobada por la experiencia lo que la convierte en una percepción positivista por la verificación positiva de los resultados.

El proyecto de tesis doctoral planteado se enfoca en su estudio a la filosofía positivista, de gran connotación en la investigación científica actualmente, porque se desarrolla a partir del planteamiento de un problema que capta la atención del investigador y es objeto de ser estudiado, observado, analizado, para determinar la fuente, causas y efectos; luego en función de los resultados productos del estudio empírico, hacer contrastaciones con otras realidades paralelas o adversas, que permitan validar los resultados y derivarlos en una propuesta coherente que siempre será positiva pues es tendiente a solucionar la situación problemática planteada.

En este acápite se han analizado las corrientes filosóficas que tienden al positivismo, pero no todos los filósofos y científicos son seguidores de esta filosofía sino por el contrario difieren de este pensamiento filosofal. A continuación, se analizarán sus puntos de vista para llegar a un entendimiento completo y profundo de los enfoques acogidos para el desarrollo del proyecto de tesis doctoral "Diseño de un Modelo de costos basados en actividades para el desarrollo del productor agrícola rural ecuatoriano".

\section{Concepción y procesos de la filosofía de las ciencias y su relación con la propuesta}

El Círculo de Viena adopta el planteamiento de Ernst Mach inclinado a que el método de la ciencia debe basarse en la inducción, donde se parte del conocimiento particular a lo general, la ciencia avanza por nuevos descubrimientos que son agregados, modelo que se desprende del positivismo lógico. Divide el estudio de la ciencia en especialidades. Determina dos métodos de validación el empírico basado en experiencias y el cognoscitivo que puede ser verificado analíticamente o experimentalmente. (Mach, 2019).

Este planteamiento es objetado por Karl Popper en su obra La lógica de la investigación científica, (1980) quien lidera el falsacionismo que descarta la inducción como método de validación de la ciencia y propone no verificar en positivo, sino, por el contrario, 
escoger una alternativa negativa que le da el carácter de falsa y rechace su existencia ya que la ciencia no avanza en función de acepciones positivas nuevas sino por el reconocimiento realista de que aquello que se considera cierto puede ser demostrado que es falso. El escepticismo forma parte de la concepción de la ciencia siendo concebida como cierta hasta que se demuestre lo contrario o sean falsadas. Es decir, la ciencia es dinámica y está en constante contrastación para ser verificada como cierta.

Imre Lakatos fortalece el enfoque popperiano porque desde su punto de vista y comparándolo con Khun, representa "la revolución científica como casos de progreso racional" ya que sostenía que la lógica es capaz de transmitir la verdad, pero no probarla. Por otro lado, sostenía que el filósofo Khun confiere que "el cambio científico de un paradigma a otro es una conversación mística que no está ni puede estar gobernada por reglas racionales a esta concepción la denominó falsacionismo metodológico ingenuo", resalta la importancia filosófica del probabilismo o neojustifiacionismo que es el resultado de la negación de una conclusión, es decir una teoría se puede falsar solo por otra teoría y no por la mera observación o experimentación. (1989).

Lakatos considera los criterios de evaluación y desarrolla la teoría de los programas de investigación científica y las definió como un conjunto de teorías relacionadas entre sí, de tal forma que unas se derivan de otras. Las evidencias surgen de varias hipótesis probadas, y los cubre de la heurística para explicar los fenómenos. (Lakatos, 1989). Al reflexionar sobre este paradigma se denota el énfasis que pone el epistemólogo en sustentar arduamente las teorías a fin de protegerlas de ser derrumbadas por otros criterios.

El proyecto de tesis si bien se acoge el positivismo como filosofía, se considera también los aspectos contradictorios específicamente en la comunidad a la que va dirigida la propuesta, ya que el perfil del agricultor rural de acuerdo a cifras del INEC tienen poca escolaridad, hay que buscar la pertinencia del modelo adecuado a su nivel educativo por lo que se entraría a la prueba de descartar diseños que resulten complejos para el sector hasta llegar a la concepción del modelo optimo en el tiempo y espacio a aplicar que cumpla la finalidad de su creación.

La Escuela de Frankfurt conformada por filósofos intelectuales de la clase pudiente de Alemania entre ellos Theodor Adorno, Walter Benjamín, Max Horkheimer, Herbert Marcuse, Jürgen Habermas, Oskan Negt o Hermann Schweppenhäuser, Erich Fromm, Albrecht Wellmer y Axel Honneth entre otros seguidores del pensamiento marxista. Se concibe que en la época de acción de esta corriente la lucha social imperaba dominada por la política, fascismo, burocratización. Esto como producto del capitalismo y la sociedad de consumo materialista que excluía a la clase obrera insatisfecha que había instaurado una industria cultural el de las masas o popular y el de masas que manipulaba a la colectividad. Esta desigualdad profunda derivó en la guerra mundial y al genocidio.

Este instituto estaba orientado al estudio de los hilos ocultos de la dominación capitalista, este compendio combinó el judaísmo con el marxismo y el humanismo con lo socialista, debido a las diferentes profesiones de sus integrantes, estos produjeron un pensamiento crítico interdisciplinario para la composición de dos conceptos: el de alienación donde el ser deja de pensar por sí mismo; y, el de manipulación donde se controla la voluntad del sujeto por el uso de los medios. Además de la Teoría Crítica que promulgaba el cambio social e histórico, donde señalan al hombre como enemigo de la naturaleza. (Wiggershaus, 1986). 
La propuesta de tesis plantea la optimización de recursos y la inclusión protagónica de un sector vulnerable de la sociedad ecuatoriana que, a pesar de su aporte a la seguridad alimentaria y economía del Ecuador, desde la época de la colonia han sido marginados con políticas que no los integran al cambio social y de progreso a la par con su contribución. En contraste los agricultores rurales han respetado sus tradiciones de cultivo logrando la conservación de la tierra y se han mantenido alejados del materialismo que predomina en la sociedad de consumo en la que coexistimos, lo que induce a pensar que se encuentran en un estado de alienación desde el punto de vista critico se debe buscar el cambio social y cambiar la historia de olvido al campesino.

\section{Los métodos de la ciencia y su relación con la propuesta de sistema de costeo para el sector agrícola rural}

A lo largo de la historia el hombre se ha visto en la necesidad de descubrir los fenómenos que lo rodean, preocupación que ha sido la constante de notables filósofos en distintos estadios en el tiempo, dedicados a dar forma a sus investigaciones en forma de ciencia no solo para dilucidar los fenómenos sino también para dar respuesta a grandes interrogantes y la creación e innovación a partir del entendimiento, es así que formulan métodos que les ayudan a organizar el conocimiento adquirido ya sea por la experiencia o el análisis, y de esta manera hacerlos comprensibles y extensibles a la sociedad.

Cada aporte teórico hecho por los hombres de ciencia en el devenir del tiempo desde Aristóteles hasta los tiempos actuales ha sido consensuado y discutidos en cada época, generando nuevas teorías o cuestionando las ya existentes, creando métodos de verificación, programas de investigación todos tendientes al desarrollo del pensamiento crítico del ser humano.

Es así que llegamos a fines del siglo XX donde irrumpe el filósofo Ilya Prigogine con su obra "El fin de las certidumbres" y revoluciona el acontecer filosofal en un sentido final positivo de las anteriores corrientes intelectuales con su teoría del caos. Sostuvo que la ciencia era un elemento de la cultura y no al contrario como lo sostiene el positivismo. Declaró que la muerte da paso a otro estado, la naturaleza es una fuente de novedades, de acuerdo a sus estudios lo estable y lo inestable son la excepción. Los cambios en la naturaleza son irreversibles. El tiempo es unidireccional y avanza inexorable, se llega al orden a partir del desorden, existe un cambio epistemológico que conlleva a nuevos desafíos. (Prigogine, 1996) Este pensamiento rompe con la alienación del ser humano, invoca al hombre a pensar más allá de su experiencia o accionar ya que situaciones anómalas puede irrumpir generando una situación caótica que generará en un nuevo conocimiento.

\section{Método}

Este estudio por su nivel es descriptivo, ya que tiene la intensión de demostrar como a través del conocimiento profundo sobre una materia en este caso la contabilidad, se pueden lograr crear metodologías viables que incluyan a sectores marginados en el panorama global de desarrollo. Por su diseño es documental con un enfoque cualitativo se ha profundizado en la revisión literaria de textos impresos y digitales de investigadores seguidores de la corriente filosofal contable y otras, que permitieron conocer de manera holística los principios, fundamentos, teorías y metodologías que rigen la disciplina, para luego de su 
entendimiento relacionarlos al diseño de herramientas contables asequibles para usuarios que no necesariamente tengan una cultura contable.

\section{Resultados}

Por medio del análisis reflexivo de los núcleos teóricos desarrollados y desde el punto de vista de lo que se desea demostrar esto es, si la aplicación de un modelo de costos basados en actividades puede ser una alternativa viable para el desarrollo del productor agrícola rural ecuatoriano, se evidencia que la concepción de la investigación, esto como base para el diseño de la herramienta contable, se rige por la corriente positivista divulgada por A. Comte, es decir la integración de los actores y recursos con que cuentan, para desarrollar metodologías afines al perfil de estos que les permita asimilar el conocimiento propuesto sin mayor esfuerzo y su posterior aplicación.

Sin embargo, la corriente positivista efectiva para el diseño no lo sería para demostrar la efectividad de la herramienta de costeo, en este punto se considera que el desarrollo de la herramienta ya concluida debe ser demostrado en falso, que según los preceptos de Karl Popper se deben aplicar métodos de validación en función de la realidad, esto es la dinámica de constante actualización a fin de que la propuesta no quede obsoleta, sino que por el contrario avance en función de la evolución de la sociedad, esto garantizaría el desarrollo sostenible del sector por su aplicación.

Queda en evidencia que si se introduce al sector agrícola rural ecuatoriano en la cultura contable a través de una herramienta pertinente con su perfil, lograran mejorar su desempeño financiero y disminuir los riesgos de pérdidas que afecten su actividad, esto acompañado de sus prácticas ancestrales serían un aporte invaluable para la sociedad del conocimiento, que se pueden transformar en teorías contables categóricas que le darían el reconocimiento ineludible de ciencia a la Contabilidad por su aporte a la colectividad, siguiendo las acepciones de Ilya Prigogine que la ciencia es un elemento de la cultura que permite al individuo pensar más allá de su experiencia y accionar generando un nuevo conocimiento.

\section{Conclusiones}

Si la verificación científica de la Contabilidad para ser reconocida como ciencia normal, está dada por la validación epistemológica de los hechos y teorías aceptadas de manera generalizada por la comunidad, queda en manos de los contadores públicos diluir a través de su inmersión en la investigación y gestión del conocimiento las brechas que obstaculizan la relación del objeto de estudio con los intereses comunes de la sociedad factor limitante para su reconocimiento, que por los diseños curriculares de pregrado no pueden ser mitigados por los profesionales contables, pero que a partir de estudios doctorales donde se involucra al doctorando a la reflexión y análisis filosófico se puede lograr el escrutinio de la epistemología de la contabilidad como una ciencia.

El sistema de costeo $\mathrm{ABC}$ estandariza cada una de las actividades del proceso productivo, mediante del consumo óptimo de recursos esto es tiempo, mano de obra, materiales; permita una aproximación real de asignación de erogaciones a cada parte del ciclo operacional. Es decir, no solo es un modelo para calcular, sino que agrega valor al sector donde se aplique, por tanto, constituye una herramienta de análisis y reflexión estratégica 
tanto para el agricultor como para los consumidores y la colectividad en general, por la reducción e incluso eliminación de explotación desmedida de patrimonios, su campo de acción va desde la planeación hasta el cierre de período económico.

Cumple entonces la condición de modelo heurístico ya que da solución al problema planteado de altos costos a reducción por su uso optimizado, en consonancia se mejora el rendimiento permitiendo el desarrollo ontológico esto es el entorno humano involucrado en el proceso. (Khuhn, 1962) Se aplica además la filosofía positivista de A. Comte considerar a todos los fenómenos como sujetos, cuyo descubrimiento preciso y la posterior reducción al menor número posible constituye la finalidad de nuestros esfuerzos. (1875).

En el panorama contextual moderno donde el bienestar de la población se liga a la capacidad de los actores para acceder al conocimiento, transformarlo, aplicarlo y aprovecharlo es imperativo incorporar al sector agrícola rural a esta dinámica de gestión del conocimiento, creando propuestas de desarrollo que sean pertinentes para los usuarios esto es los productores rurales, es decir diseñadas acorde con su perfil.

\section{Bibliografía}

Alarcón, E. (27 de Enero de 2014). vidauniversitaria. (U. d. Navarra, Productor) Recuperado el 24 de Marzo de 2019, de https://www.unav.edu: https://www.unav.edu/web/vidauniversitaria/detallenoticiapestania/2014/01/27/tom\%C3\%A1s-de-aquino-y-lafilosof\%C3\%ADa?articleId=3736078

Atencia, J. M. (1991). Positivismo y Neopositivismo. Anales del Seminario de Metafísica. 25, págs. 143-154. Madrid: Universidad Complutense. Obtenido de file:///D:/_Usuarios/ERIKA/Downloads/18631-18707-1-PB.PDF

Baltodano, A. V. (2010). Filosofía de las Ciencias para la creación de conocimientos (Primera ed.). Lima, Perú: San Marcos de Aníbal Paredes Galván. Recuperado el 10 de Febrero de 2013

Barton, P. R. (18 de Abril de 2018). El pensamiento y la personalidad de William James. México: W 4JUxi] KoOopá . Obtenido de coebiotica.salug: http://coebioetica.saludoaxaca.gob.mx/wp-content/uploads/2018/libros/ceboax-0524.pdf

Comte, A. (1875). Principio de Filosofía Positiva. (J. Lagarrugue, Trad.) Santiago: Imprenta de la Libreria del Mercurio. Recuperado el 25 de Marzo de 2019, de file:///D:/_Usuarios/ERIKA/Downloads/principios-de-filosofia-positiva.pdf

Diaz , I. M. (2003). La Contabilidad Social-Origen y paradigmas. Revistas investigación QUIPUKAMAYOC, 31-42. Recuperado el 23 de Marzo de 2019, de http://revistasinvestigacion.unmsm.edu.pe/index.php/quipu/article/viewFile/5513/474 9

Gómez, D. R. (10 de Enero de 2019). elproductor.com. Recuperado el 25 de Marzo de 2019, de elproductor.com: http://elproductor.com/editorial-del-mes/el-sector-agropecuariobalance-2018/

Goode, W. J., \& Hatt, P. K. (1975). Métodos de investigación social. México: Trillas.

Hedegger, M. (1993). Ser y Tiempo (Primera ed.). (C. J. Rivera, Trad.) Santiago de Chile, Chile: Editorial Universitaria S. A. Recuperado el 25 de Marzo de 2019, de file:///D:/_Usuarios/ERIKA/Downloads/822.pdf

Khuhn, T. S. (1962). The Structure of scientific (Primera ed.). (C. Agustín, Trad.) Chicago, Estados Unidos: University of Chicago Press. Recuperado el 23 de Marzo de 2019, de https://www.google.com/search?ei=E2eZXIDgHqHZ5gKt9pCIBg\&q=estructura+de+ 
la+revoluciones+cientificas+thomas+kuhn\&oq=Estructura+de+la+Revoluciones+Cie nt\%C3\%ADficas\&gs_l=psy-

ab.1.0.0i22i3015.2072.2072..5458...0.0..0.186.186.0j1_....0...2j1..gws-wiz.B0XhrNK

Lakatos, I. (1989). La metodología de los programas de investigación científica. (J. Worall,

G. Currie, Edits., \& J. C. Zapatero, Trad.) Madrid, España: Alianza Editorial.

Recuperado el 23 de Marzo de 2019, de

https://epistemologiaufro.files.wordpress.com/2010/10/lakatos.pdf

Lyotard, J.-F. (1994). La posmodernidad explicada a los niños (Tercera ed.). (E. Linch,

Trad.) Galice, París, Francia: Gedisa. Recuperado el 25 de Marzo de 2019, de

https://kupdf.net/download/la-posmodernidad-explicada-a-los-nios-jean-francoislyotardpdf_59f1f041e2b6f52d5e54ffd7_pdf

Mach, E. (23 de Marzo de 2019). bibliotecadigital.ilce.edu.mx. Obtenido de

bibliotecadigital.ilce.edu.mx:

http://bibliotecadigital.ilce.edu.mx/sites/ciencia/volumen3/ciencia3/161/html/sec_34.h tml

Montes, S. C., Soto, M. E., \& Valencia, S. J. (2006). Paradigmas en Contabiidad (Primera ed.). (A. G. Ltda., Ed.) Cali, Colombia: Artes Gráficos Ltda. Recuperado el 22 de Febrero de 2019, de http://eutimiomejia.com/portal/images/textos/pdf/LIBRO_PARADIGMA_EN_CONT ABILIDAD.pdf

Popper, K. R. (1980). La lógica de la investigación científica. (D. V. Sánchez, Trad.) Madrid: Tecnos. Recuperado el 23 de Marzo de 2019, de http://psikolibro.blogspot.com

Prigogine, I. (1996). La fin des certitudes. Chile: Andrés Bello. Recuperado el 23 de Marzo de 2019, de medicinayarte.com: http://medicinayarte.com/img/el-fin-de-lascertidumbres.pdf

Spirkin, A. G. (1969). Materialismo dialéctico y lógica dialéctica (Primera ed.). (J. Lain, Trad.) Mexico, México: Editorial Grijalbo. Recuperado el 23 de Marzo de 2019, de file:///D:/_Usuarios/ERIKA/Downloads/materialismo-dialectico-y-logicadialectica.pdf

Torres, G. A. (Octubre de 2012). La fislosofia de la ciencia contable. Quipukamayoc, 20(38), 32-49. Recuperado el 23 de Marzo de 2019, de http://revistasinvestigacion.unmsm.edu.pe/index.php/quipu/article/viewFile/4422/351 1

Wiggershaus, R. (1986). La Escuela de Fráncfort. (H. M. Romano, Trad.) Titivillus.

Recuperado el 23 de Marzo de 2019, de http://ceiphistorica.com/wpcontent/uploads/2016/04/Wiggershaus-Rolf-La-escuela-de-Francfort-.pdf 